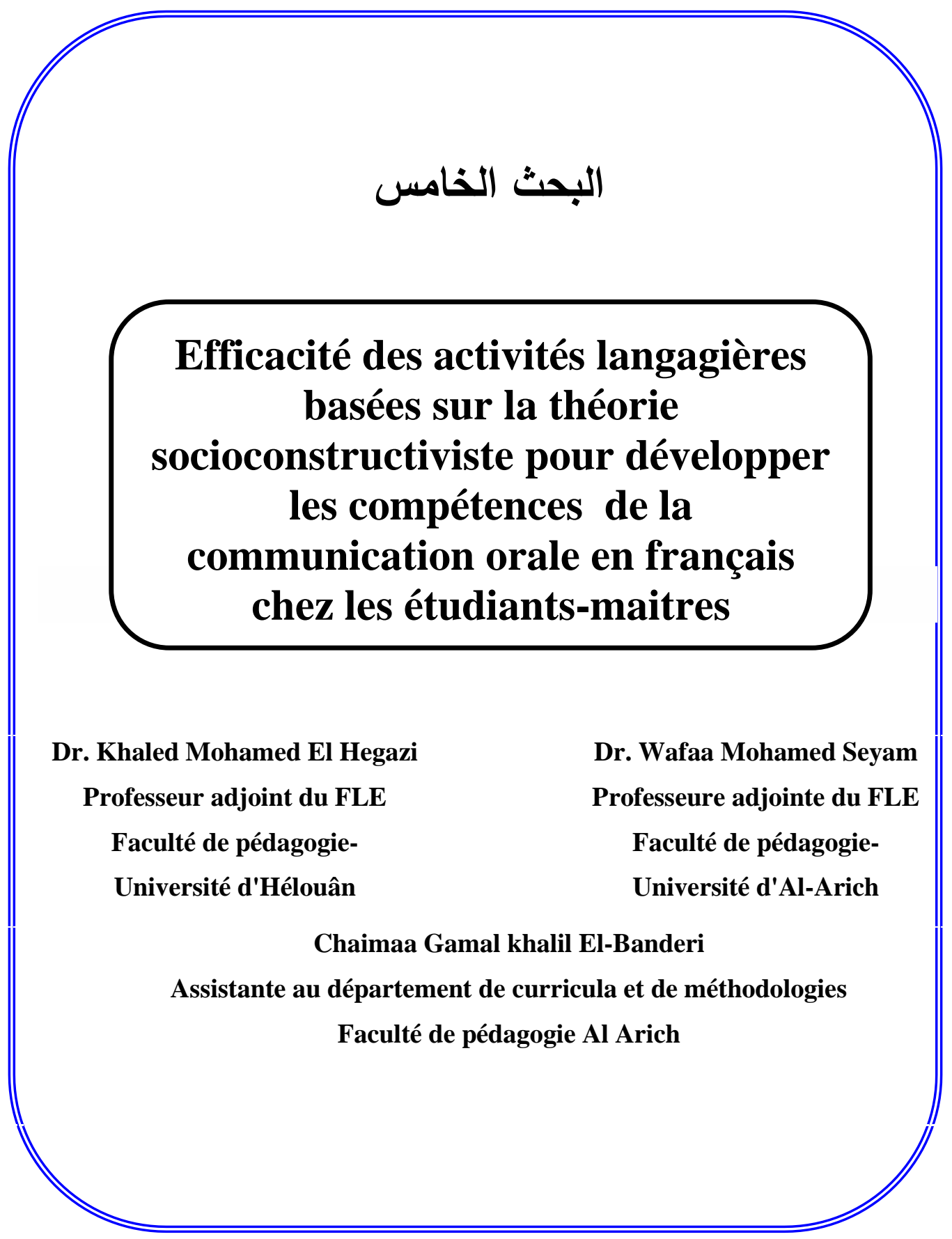


Efficacité des activités langagières basées sur la théorie socioconstructiviste pour développer les compétences de la communication orale en français chez les étudiants-maitres

Dr. Khaled Mohamed El Hegazi, Dr. Wafaa Mohamed Seyam, Chaimaa Gamal khalil El-Banderi 


\section{Efficacité des activités langagières basées sur la théorie socioconstructiviste pour développer les compétences de la communication orale en français chez les étudiants-maitres}

Dr. Khaled Mohamed El Hegazi

Professeur adjoint du FLE

Faculté de pédagogie- Université d'Hélouân
Dr. Wafaa Mohamed Seyam

Professeure adjointe du FLE

Faculté de pédagogie- Université d'Al-Arich

\section{Chaimaa Gamal khalil El-Banderi}

Assistante au département de curricula et de méthodologies

Faculté de pédagogie Al Arich

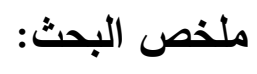

تناول هذا البحث استخدام أنشطة لغوية قائمة علي النظرية البنائية الاجتماعية في

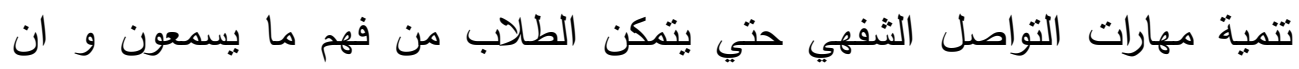

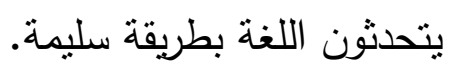

من خلال خبرة الباحثة الثخصية و اعطاء سكاشن لطلاب الكلية و الاشراف علي مجموعات التربية العطلية في المدارس تبين وجود ضعف في بعض مهارات

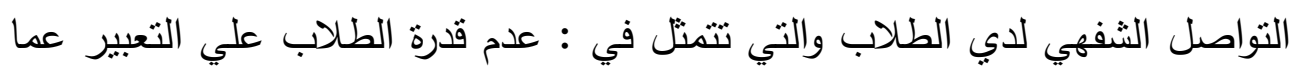

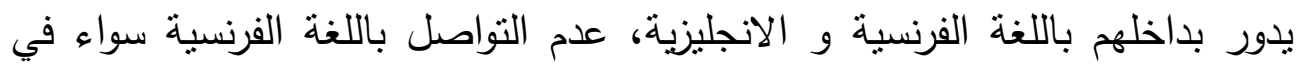
التربية العملية او في التدريس المصغر.

و يقصد بأنشطة لغوية قائمة علي النظرية البنائية الاجتماعية ، انها مجموعة من

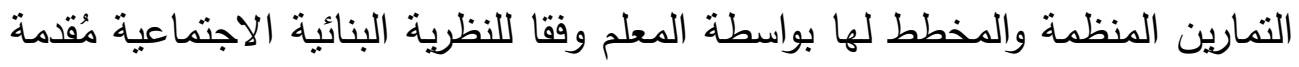


للطلاب لاكتشاب بعض مهارات التواصل الثفهي (الاستماع ، التحدث )، ولضبط جميع المتغيرات تم رصد و تقييم نتائج الطلاب في الاختبار القبلي والبدي، و ذللك من خلال تحليل و تفسير البيانات و النتائج بطريقة كمية و كيفية، و اتبعت الباحثة في ذللك المنهج شبه التجريبي. كلمات مفتاحية : أنشطة لغوية ، البنائية الاجتماعية ، التواصل الثفهي.

\section{Résumé}

Cette recherche porte sur l'efficacité des activités langagières basées sur la théorie socioconstructiviste pour développer les compétences de la communication orale et pour aider les apprenants de parler facilement et directement.

Durant le stage pratique et les cours de didactique du FLE, la chercheuse remarque que les apprenants de $2^{\text {eme }}$ année à la faculté de pédagogie ne possèdent pas les compétences de la communication orale en français (compréhension - production ) à travers les travaux hebdomadaires avec eux.

Rappelons que les activités langagières basées sur la théorie socioconstructiviste sont comme des taches authentiques basant sur la réception et la production qui conduisent l'apprenant à écouter afin de réagir et s'exprimer oralement. Elle a surveillé et évalué les notes des apprenants au pré/ post test d'une manière quantitative et qualitative en suivant la méthode semi-expérimentale.

Mot clés : activités langagières, socioconstructiviste, communication orale.

Introduction:

Avec les progrès réalisés dans tous les domaines de la vie, personne ne peut nier l'importance des langues étrangères. Le but de l'enseignement de ces dernières n'est pas uniquement culturel, il est également pratique: la maîtrise d'une langue étrangère est 
équivalent à la possession d'un outil de communication internationale.

Apprendre une langue est acquérir une certaine compétence de communication dans cette langue ce dont l'apprenant a besoin d'être capable de communiquer en langue étrangère, connaître ses règles linguistiques ainsi que ses règles d'usage afin qu'il les adopte aux différentes situations de communication. Pour maîtriser la communication en langue étrangère, on doit développer les deux compétences à savoir : la compréhension orale et la production orale.

En effet, la langue, en tant que moyen de communication, c'est avant tout une langue parlée. La fonction du langage est de permettre aux individus de communiquer entre eux, c'est- à- dire de se parler. Autrement dit, la réalité vivante d'une langue est la communication orale. (Sayed. N.Z,2008)

De surcroit, la communication est la finalité essentielle de l'apprentissage d'une langue étrangère car c'est en parlant qu'on apprend à communiquer. Vu qu'une " langue est d'abord une réalité orale " ( Procher. L, 2004), l'enseignement de l'oral est une nécessité dans l'apprentissage du FLE.

Plane, S (2004) propose un cadre général d'analyse pour pouvoir identifier les différentes fonctions didactiques de l'oral dans la classe qui

1- L'oral, moyen d'expression: l'expression orale favorise le développement personnel et la construction d'une identité sociale. Il s'agit de favoriser l'expression personnelle et d'encourager la parole de l'apprenant.

2- L'oral, moyen d'enseignement: l'oral (du maître) sert à la transmission d'informations et à la régulation pédagogique, comme par exemple expliciter un point grammatical précis ou contrôler les comportements des apprenants. Dans ce caslà, la parole de l'enseignant sert à réguler la classe et à transmettre des informations. 
3- L'oral, objet d'apprentissage: les apprenants peuvent apprendre à communiquer, maîtriser la langue orale.

Dans cette perspective et à travers la pratique d'exercices variés, il s'agira de travailler des compétences aussi bien communicationnelles (s'exprimer, interagir, etc. ) que langagières orales (répondre aux questions, manifester son accord, son désaccord, etc.).

4- L'oral, moyen d'apprentissage: les apprenants apprennent par la verbalisation et par les interactions. C'est un oral considère à la fois comme un savoir mais aussi comme un medium d'apprentissage.

5- L'oral , objet d'enseignement: il est possible de faire du travail de verbalisation et d'interaction un objet d'enseignement . Dans ce cas, l'oral est considère, tout comme la lecture, comme un objet d'enseignement à part entière.

Le défi majeur, en ce qui a trait à la communication orale, consiste à outiller les apprenants qu'ils deviennent des auditeurs avisés et qu'ils soient en mesure de prendre la parole de manière efficace dans un grand divisé de situations.

La communication orale fait l'objet de recherches, visant à montrer la nécessité de son enseignement et de son apprentissage.

L'étude de ( El-Adl- Ahmed, 2002) qui vise à connaître l'influence d'un programme d'activités langagières proposé en vue de développement de l'expression orale chez les étudiants des facultés de pédagogie, l'étude de (Hagar Khalil, 2005) qui vise à connaître l'effet d'un programme d'employer le Brain Storming sur le développement des compétences de l'expression orale en français chez les étudiants des écoles secondaires des langues, et l'étude de (Nglaa Fathi , 2006) qui propose de connaître l'effet d'un programme propose base sur le jeu de rôle dans l'enseignement de la langue français pour développer quelque 
compétences de la communication orale chez les étudiants de la première année a la faculté de Minia.

L'étude de ( Abd el Moniem Samir, 2014) qui connaît l'effet d'un programme proposé basé sur l'approche constructiviste pour développer quelque compétences de l'expression orale fonctionnelle en français chez les étudiants des instituts supérieurs du tourisme de l'hôtellerie, l'étude de ( Rania Salah, 2007 ) qui vise à connaître l'efficacité d'utilisation de la dramatisation créative sur le développement des compétences de l'expression orale chez les étudiants du département de français à la faculté de pédagogie

Ces études confirment que les compétences de la communication orale sont des compétences très importantes que les apprenants doivent apprendre, car cela reflète sur le niveau langagière de leurs apprenants.

Pour cela, on doit s'occuper de chercher de méthodologies nouvelles basées sur l'apprenant où il est considère comme autonome et appuyé par des ressources authentiques adaptés à ses besoins dans un processus coopératif et où tout est centré sur lui. Il faut diversifier les moyens d'enseignements utilisés pour développer les compétences de la communication orale.

Le constructivisme se base sur la théorie de Piaget (le constructivisme cognitif) et la théorie de vygotsky ( le constructivisme social ). La pensée constructiviste a manifesté dans l'œuvre de Piaget et vygotsky qui confirment que le changement cognitif se produit avec le déséquilibre entre les concepts antérieurs et les nouvelles connaissances. On remarque que le socioconstructivisme qui se bonde au constructivisme met l'accent sur le rôle des interactions sociales multiples dans la construction des savoirs.

La théorie socioconstructiviste joue un rôle principal à l'interaction sociale basée sur la communication entre l'individuel et la société, et confirme de l'enseignement individuel ou social. Cela manifeste le rôle de la théorie socioconstructiviste en vue de 
développer les compétences de la communication orale. (Learman, S , 2000)

L'efficacité d'un processus d'apprentissage réside sans aucun doute dans la qualité des contenus transmis au cours des activités de classe, et dans la qualité des procédés mis en œuvre pour faire comprendre, assimiler et rendre accessibles ces contenus. Pour arriver à tout cela, les activités langagières seront un outil très puissant, car elles prennent l'attention du processus didactique même, et permettent un investissement plus rentable. Les activités permettent de renforcer la motivation puis présentent par l'acquisition de nouvelles connaissances qui mettent en pratique celles qui sont déjà acquises.

Plusieurs recherches ont confirmé que l'utilisation de la théorie constructiviste et socioconstructiviste dans l'enseignement et l'apprentissage du FLE particulièrement l'étude de (Hala Ahmed, 2017) qui vise l'efficacité d' un dispositif numérique proposé selon un paradigme socioconstructiviste pour développer les compétences de la production orale et écrite chez les étudiants de FLE à la faculté de pédagogie de Quena, l'étude de (Mohamed Fathy, 2009) qui vise l'utilisation un modèle constructiviste pour apprendre les compétences de l'expression écrite chez les élèves du cycles secondaire, ces études ont recommandé l'utilisation de ces stratégies (l'apprentissage par problèmes et l'apprentissage par projet ) en vue de développer les compétences de la communication orale à travers les activités collaboratives.

Pour le socioconstructivisme, la dimension du contact avec les autres afin de construire les connaissances. La construction d'un savoir, bien que personnelle, s'effectue dans un cadre social. Les informations sont en lien avec le milieu social. Le socioconstructivisme privilégie le rôle actif de l'apprenant dans le processus de construction des connaissances. (Lafortune. L \& Deaudelin. C, 2001).

Dans la théorie socioconstructiviste, l'apprentissage est perçu comme un processus autonome dans lequel les initiatives 
sont acceptées et même encouragées, et aussi les facteurs sociaux et cognitifs interagissent pour conduire à une organisation mentale plus évoluée, construisant et développant ainsi les théories el les compétences.

De ce qui précède, on manifeste que la théorie socioconstructiviste donne une grande importance à l'activité de l'apprenant dans la construction de son savoir. Cet apprenant compare, organise, transforme et assimile les informations et les données par reconstruction à sa structure conceptuelle. Elle convient aux compétences de la communication orale. De plus, la théorie socioconstructiviste est une approche qui, du point de vue de la chercheuse, n'est pas utilisée en vue de développer la communication orale de la langue française en Égypte.

Pour cela, la chercheuse essaye de développer les compétences de la communication orale chez les étudiantsmaitres à la faculté de pédagogie par l'application d'une unité expérimentale basée sur des activités langagières basées sur la théorie socioconstructiviste.

\section{1- Sensibilisation au problème de la recherche:}

on a affronté au problème de la recherche travers les ressources suivantes :

1- le stage pratique et les cours de didactique du FLE, la chercheuse a remarqué que les apprenants de $2^{\text {eme }}$ année à la faculté de pédagogie ne possèdent pas les compétences de la communication orale en français (compréhension - production) à travers les travaux hebdomadaires.

2- D'autre part, il existe un nombre d'études qui ont montré la faiblesse des apprenants à la faculté de pédagogie en communication orale: compréhension, production. Parmi ces recherches ; l'étude de (Hamada Abou Elftouh, 2013) qui vise l'utilisation de l'approche interculturelle pour développer les compétences de la communication orale chez les élèves du cycle 
préparatoire, l'étude de ( Hanan Hafez,2009) qui vise l'évaluation du traitement didactique de la communication orale en français chez les futurs enseignants dans les facultés de pédagogie à la lueur de l'enseignement stratégique, l'étude de (Marina Mikhail, 2011) qui a développé les compétences de l'expression orale en utilisant la stratégie de l'enseignement réciproque chez les élèves du cycle secondaire, l'étude de (Névine Mahmoud, 2008) qui vise l'efficacité d'un programme basé sur l'apprentissage coopératif pour développer les compétences de l'expression orale chez les futurs enseignants et l'étude de ( Amr Kammoun,2012) qui vise le développement les compétences de l'expression orale en langue française, deuxième langue étrangère, au cycle préparatoire en utilisant la stratégie proposée basée sur l'approche interactionnelle.

3- La majorité des apprenants de la deuxième année ne s'expriment pas aisément en langue française ils éprouvent des difficultés à l'oral : stock lexical restreint, mauvaise prononciation, mécompréhension du langage, difficulté en expression,... etc

4- Les activités exploitées ne prennent aucunement en considération les principes de nouvelles approches qui suggèrent d'introduire dans la classe des documents authentiques. Il y a un manque du travail en groupe, d'exposés oraux, des jeux de rôles, des scènes de théâtre, ...etc, qui peuvent présenter de bonnes situations pour améliorer les compétences de la communication orale de nos apprenants

5- Les entrevues avec les professeurs au département de la langue française pour connaître le niveau actuel de ces apprenants à la faculté de pédagogie à Al-Arich en compétences de la communication orale. Ces professeurs se mettent d'accord sur le fait qu'il y a une 
faiblesse en compétences de la communication orale chez les apprenants à la faculté de pédagogie.

\section{2- Problématique de la recherche:}

Le problème de cette recherche réside en faiblesse en compétences de la communication orale auprès des étudiants maitres à la faculté de pédagogie.

Cette recherche a donc essaye de répondre à la question principale suivante:

Quelle est l'efficacité des activités langagières basées sur la théorie socioconstructiviste pour développer les compétences de la communication orale en français chez les étudiants- maîtres à la faculté de pédagogie?

Dans cette perspective, la chercheuse essaie de répondre aux sous-questions suivantes:

- Quelles sont les compétences de la communication orale en français nécessaires aux étudiants - maîtres à la faculté de pédagogie?

- Quelle est la forme proposée d'une unité qui comprend des activités langagières basées sur la théorie socioconstructiviste pour développer les compétences de la communication orale en français chez les étudiants - maîtres à la faculté de pédagogie?

- Quelle est l'efficacité d' une unité qui comprend des activités langagières basées sur la théorie socioconstructiviste pour développer les compétences de la communication orale en français chez les étudiants - maîtres à la faculté de pédagogie ?

\section{3- Objectif de la recherche:}

Cette recherche a pour objectif de vérifier l'efficacité des activités langagières basées sur la théorie socioconstructiviste pour développer les compétences de la communication orale en français chez les étudiants-maitres.

Provient de cet objectif principal à discuter: 
- Développer les compétences de la communication orale en français chez les étudiants - maîtres à la faculté de pédagogie.

- Expérimenter l'efficacité d'une unité qui comprend des activités langagières basées sur la théorie socioconstructiviste pour développer les compétences de la communication orale en français chez les étudiants maîtres.

\section{4- Délimitations de la recherche:}

Cette recherche se limite à

1- Un échantillon d'étudiants - maîtres en 2eme année à la faculté de pédagogie, à l' université d' Al-Arich.

2- Certaines compétences de la communication orale.

3- Des activités langagières basées sur la théorie socioconstructiviste.

4- Une unité basée sur des activités langagières basées sur la théorie socioconstructiviste.

\section{5- Hypothèses de la recherche:}

Cette recherche vise à examiner les hypothèses suivantes :

1- Il n'existe pas de différence statistiquement significative au niveau de (0.01) entre les moyennes des notes des étudiants - maitres du groupe expérimental et du groupe témoin aux pré - tests, en ce qui concerne les compétences de la communication orale (compréhension - production).

2- Il existe une différence statistiquement significative au niveau de (0.01) entre les moyennes des notes des étudiants - maitres du groupe expérimental et du groupe témoin aux post - tests, en ce qui concerne les compétences de la communication orale (compréhension - production ) en faveur du groupe expérimental. 
3- Il existe une différence statistiquement significative au niveau de (0.01) entre les moyennes des notes des étudiants - maitres du groupe expérimental aux pré/post tests, en ce qui concerne les compétences de la communication orale (compréhension - production ) en faveur du post - test.

4- Les activités langagières basées sur la théories de la socioconstructiviste ont un effet positif sur le développement des compétences de la communication orale chez les étudiants-maitres à la faculté de pédagogie.

\section{5- Outils et matériels de la recherche :}

Pour atteindre les objectifs de cette recherche, la chercheuse a préparé et a utilisé les outils suivants:

1- une liste des compétences de la compréhension et de la production orales.

2- Un test des compétences de la compréhension orale et de la production orales.

3- Des activités langagières basées sur la théorie socioconstructiviste pour développer les compétences de la communication orale.

4- Une unité basée sur des activités langagières basées sur la théorie socioconstructiviste.

\section{6- Méthodologie de la recherche:}

Le plan de l'expérimentation sera type " pré-post tests avec des deux groupes (expérimental - témoin )", pour examiner des activités langagières basées sur la théorie socioconstructiviste pour développer les compétences de la communication orale en français chez les étudiants - maîtres à la faculté de pédagogie. 


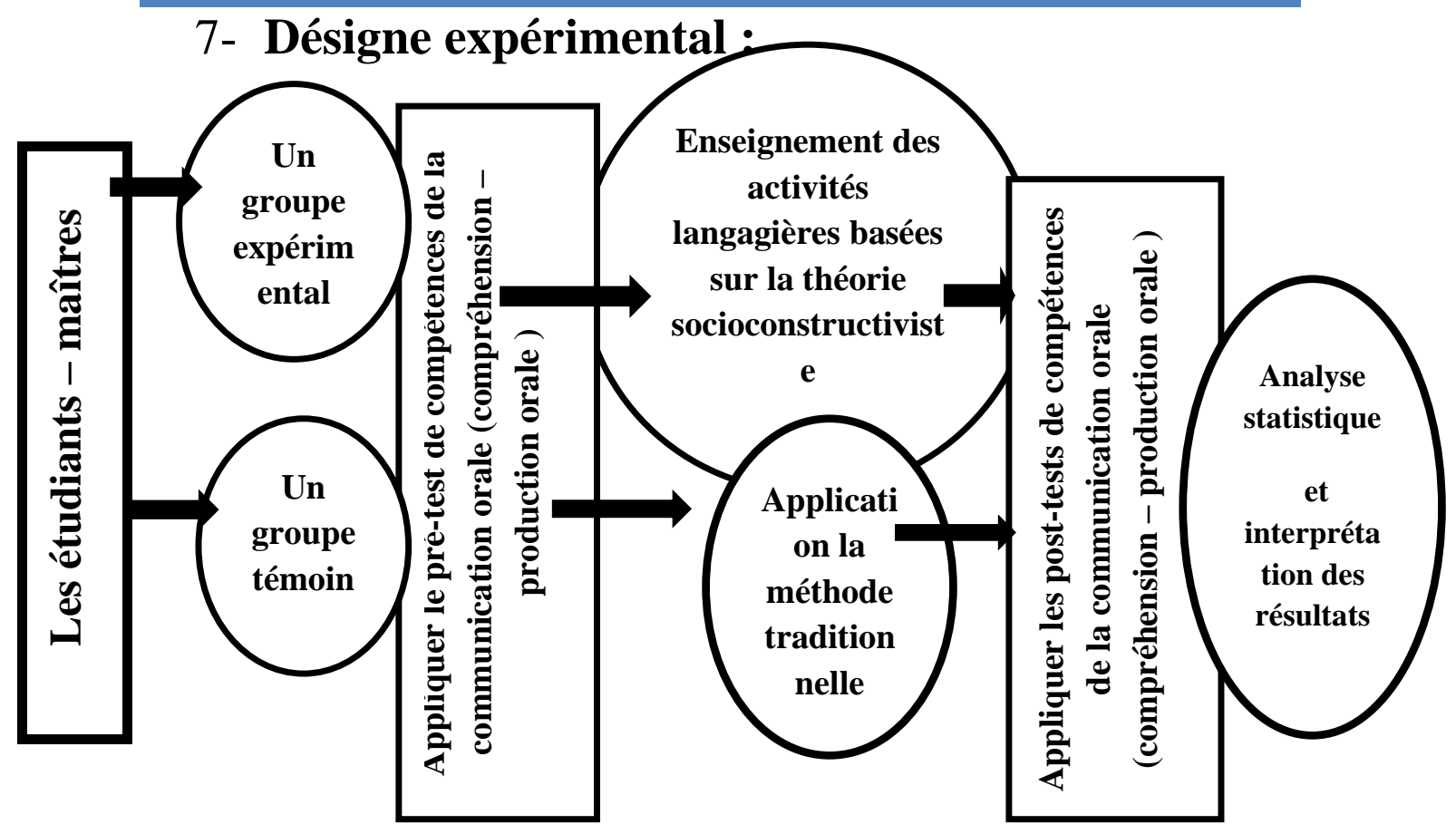

Modèle (1) la planification expérimentale

\section{8- Procédures de la recherche :}

Pour répondre aux questions de cette recherche et pour vérifier les hypothèses de la recherche, la chercheuse va suivre les étapes suivantes:

1- Pour répondre à la première question: Quelles sont les compétences de la communication orale nécessaires aux étudiants - maîtres à la faculté de pédagogie ? on va suivre les étapes suivantes:

- Consultation des recherches et des études antérieures élaborées dans le domaine de communication orale, et la théorie socioconstructiviste.

- Préparer une liste de compétences de la communication orale qui est nécessaire pour les étudiants- maîtres à la faculté de pédagogie.

- Présentation de la liste aux membres du jury pour vérifier leurs opinions. 
- Parvenir à la forme finale de la liste et faire les modifications des membres du jury.

2- Pour répondre à la deuxième question: Quelle est la forme des activités langagières basées sur la théorie socioconstructiviste pour développer les compétences de la communication orale chez les étudiants - maître à la faculté de pédagogie ?

On a suivi les étapes suivantes:

- Consultation des recherches et des études antérieures élaborées dans la théorie socioconstructiviste.

- Construction des activités langagières à la lumière de la théorie socioconstructiviste.

- Choisir les stratégies convenables de la théorie socioconstructiviste.

- Présentation l'unité basée sur des activités langagières aux membres du jury pour vérifier leurs opinions.

3- Pour répondre à la troisième question: Quelle est l'efficacité des activités langagières basées sur la théorie socioconstructiviste pour développer les compétences de la communication orale chez les étudiants - maîtres à la faculté de pédagogie ?

- Préparer des tests des compétences de la communication orale (la compréhension, la production)

- Présentation des tests aux membres du jury pour vérifier leurs opinions.

- Parvenir à la forme finale des tests et les modifications des membres du jury.

- Choix de l'échantillon de la recherche de la 2eme année à la faculté de pédagogie à Al-Arich.

- Application des pré-tests sur les deux groupes (expérimental, et témoin)

- Enseignement l'unité basée sur des activités langagières au groupe expérimental à la lueur de la théorie 
socioconstructiviste ( apprentissage par problèmes apprentissage par projet ).

- Application des post-tests sur les groupes de la recherche.

- Analyse statistique et interprétation des résultats.

- Discuter et analyser les résultats.

- Présentation des recommandations et des suggestions.

\section{0-Terminologies de la recherche:}

\section{Le socioconstructivisme :}

(Crahay, M, 1999) définit le socioconstructivisme comme une théorie qui postule que l'apprenant élabore et établit des schèmes assimilateurs dont la progression et le développement impliquent une dynamique d'équilibration. Dans la plupart des cas l'apprenant n'agit pas seul sur le réel, il a plutôt l'occasion de coordonner ses propres actions avec d'autrui afin d'enrichir ses opérations.

\section{L'activité langagière:}

Selon le Cadre Européen Commun de Référence pour Les Langues (CECR,2001, p.10), les activités langagières impliquent l'exercice de la compétence à communiquer langagièrement, dans un domaine déterminé pour traite (recevoir et/ ou produire ) un ou des textes en vue de réaliser une tâche.

Dans cette recherche, la chercheuse peut définir les activités langagières comme un groupe d'activités planifiées et gérées par les étudiants- maitres à la $2^{\text {eme }}$ en utilisant la langue sous la direction de l'enseignant en classe de langue individuellement ou par groupe. Ces activités sont pratiquées dans les différentes étapes du processus de la communication orale ( compréhension- production ) en employant plusieurs processus mentaux comme la comparaison, la mémorisation, 
l'interaction, ce qui aide à développer leurs compétences de la communication orale (compréhension - production ), à réaliser les objectifs d'apprentissage et à élaborer de nouvelles connaissances.

\section{la communication orale:}

(Giffard, D, 1985: 4), définit la communication comme un conduit psychologique pour transformer les informations à travers d'utiliser la langue, les situations, et les gestes.

Un rapport interactif entre émetteur et destinataire qui faut appel à une capacité de comprendre l'autre, produire des énoncés à l'oral dans toutes situations de communication.

La communication orale pour ( Estelle, D,2003), c'est un échange qui implique un versant " compréhension" ( écouter ) dont le récepteur est l'appareil auditif et un versant " expression " ( parler ) dont l'émetteur est l'appareil phonatoire.

La chercheuse peut définir la communication orale comme la capacité de l'apprenant à avoir des interactions linguistiques appropriées pour comprendre et exprimer dans toutes les situations de la vie quotidienne.

Après cette présentation de la problématique de la recherche, ses questions, ses objectifs, son importance, ses délimitations, ses hypothèses, ses outils, sa méthodologie, ses procédures et ses terminologies qu'on a expliqués dans ce chapitre, on va parler, dans le chapitre suivant, du cadre théorique de cette recherche. Ce cadre porte sur deux parties qui sont

A) la théorie de la socioconstructiviste et ses stratégies

B) les compétences de la communication orale et les activités langagières.

\section{1- Analyse qualitative des résultats:}

D'après les tableaux ci-dessus, on a quelques remarques :

A) Pour la compréhension et la production (communication orale ) des apprenants : 
- Les résultats des pré-tests en compréhension orale de deux groupes (expérimental - témoin) ont montré la faiblesse des apprenants en deuxième année à la faculté de pédagogie, université d' Al-Arich, la moyenne faible des notes aux pré-tests de la compréhension orale $(13,000)$, a affirmé le niveau médiocre de la compréhension, c'est pour quoi, on a remarqué que les apprenants n'ont pas la capacité d'écouter dans situation de communication orale et s'il y a un petit nombre d'apprenants qui veut participer à la communication orale, ils commettent des fautes d'identifier les sujet principal, de déterminer les idées principales, de comprendre le contenu et de résumer le discours oral.

- La même chose pour la production orale, la moyenne des notes est faible $(13,000)$ et cela montre le niveau productif médiocre et signifie aussi l'incapacité de s'exprimer et d'entrer en dialogue avec les autres dans des situations de communication orale.

B) Nous pourrions interpréter l'efficacité des activités langagières basées sur la théorie socioconstructiviste par plusieurs raisons :

1- La conception de tâches réelles ou simulées de la vie réelle attire l'attention des apprenants vers l'utilité de l'apprentissage ce qui les incite à participer à la réalisation de l'activité.

2- L'intégration entre les activités langagières aide les apprenants à se représenter la langue comme un tout. Cette perception les rend capables lorsqu'ils passant à la production orale d'utiliser les informations et de mettre en œuvre les compétences acquises au cours de la compréhension orale.

3- L'authenticité des documents autour desquels sont organisées les différentes activités langagières fait entrer la vie en classe de langue. De même, ces documents servent de modèle lorsque les apprenants passent à la production orale. 
4- Les différentes activités langagières aident au développement de l'autonomie car elles donnent un rôle actif aux apprenants dans leur apprentissage et laissent aussi plus de place au travail en groupe, à l'initiative et à la créativité.

\section{2- Recommandations de la recherche :}

Lors de nos observations et les résultats obtenus tout au long de la recherche, la chercheuse recommande de :

1. S'intéresser à faire acquérir et à développer les compétences de la communication orale du FLE aux différents cycles éducatifs.

2. Centrer sur l'apprenant, sur ses besoins et ses intérêts pour développer sa confiance en lui- même et pour le rendre de plus autonome dans la construction de ses savoirs.

3. Prendre en considération les recommandations du cadre Européen commun de Références pour les langues qui constitue une approche totalement nouvelle dont le but est de repenser les objectifs et les méthodes d'enseignement des langues pour fournir une base commune pour la conception des programmes, de diplômes et de certificats.

4. La mise à la disposition des professeurs un large éventail d'activités langagières efficaces répondant mieux aux besoins et aux intérêts des différents style d'apprentissage des apprenants.

5. Emploi des approches, des méthodes et des stratégies mettant en lumière le rôle actif des apprenants dans la construction personnelle de leurs connaissances.

6. S'intéresser à développer les compétences sociales à côté des compétences cognitives et linguistiques.

7. Inciter les enseignants du FLE aux autres méthodes d'enseignement lors de leurs parcours professionnel.

\section{3- Suggestions de la recherche:}


Complétant les résultats de la recherche et les recommandations, la chercheuse présente ses suggestions qui peuvent être traitées dans d'autres recherches.

1. Etudier l'efficacité de l'approche interactionnelle sur le développement d'autres compétences langagières chez les apprenants des facultés de pédagogie.

2. Utiliser la technologie de l'information et de la communication pour développer les compétences de la communication orale et écrite dans des différents cycles éducatifs.

Effectuer des recherches basées sur la théorie socioconstructiviste et ses stratégies pour développer les autres compétences langagières.

\section{1- Références en langue française:}

\section{A) Thèses en langue française :}

1- Abdallah, Hala . (2017). Dispositif numérique proposé selon un paradigme socioconstructiviste pour développer les compétences de la production orale et écrite chez les étudiants de FLE à la faculté de pédagogie de Quena, thèse de doctorat, faculté de pédagogie de Quena, université du sud de la vallée.

2- Abd-El Rihim, Rania. (2007). Efficacité d'utilisation de la dramatisation créative sur le développement de compétences de l'expression orale chez les étudiants du département de français à la faculté de pédagogie .Thèse de magistère. Faculté de pédagogie , Université de Zagazig

3- Abou Elftouh, Hamada .(2013). Utilisation de l'approche interculturelle pour développer les compétences de la communication orale chez les élèves du cycle préparatoire, faculté de pédagogie, thèse de magistère, université d'Ain chams.

4- Abou Shanab, Mohamed . (2009). Un modèle constructiviste pour appendre les compétences de l'expression écrite chez les 
élèves du cycle secondaire. thèse de magistère, faculté de Hélouân.

5- Al Saka, Abdel monème .(2014). Effet d'un programme proposé basée sur l'approche constructiviste pour développer quelques compétences de l'expression orale fonctionnelle en français chez les étudiants des instituts supérieurs du tourisme de l'hôtellerie .Thèse de magistère. Faculté de pédagogie , université de Ménoufya.

6- El mahdi, El Adl.(2002). Programme d'activités langagières proposé en vue de développement de l'expression orale chez les étudiants des faculté de pédagogie. Thèse de magistère . faculté des jeunes de filles, université d'Ain Chams

7- Gadalla, Hagar.(2005). Effet d'employer le brainstorming sur le développement des compétences de l'expression orale en français chez les étudiants des écoles secondaires des langues - thèse de magistère .faculté de pédagogie, université de Zagazig

8- Kammoun, Amr .( 2012). Stratégie préposée basée sur l'approche interactionnelle en vue de développer les compétences de l'expression orale en langue française, deuxième langue étrangère, au cycle préparatoire. Institut d'études pédagogiques, thèse de magistère, université du Caire.

9- Mahmoud, Névine . ( 2008). Efficacité d'un programme basé sur l'apprentissage coopératif pour développer les compétences de l'expression orale chez les futurs enseignants, faculté de pédagogie, thèse de magistère, université d'Ain chams.

10- Mikhaïl, Marina . (2011). Efficacité de la stratégie de l'enseignement réciproque pour le développement des compétences de l'expression orale chez les élèves du cycle secondaire . thèse de magistère . faculté de pédagogie , université d'Ain Chams

11- Mohamed, Naglaa . (2006) . L'effet d'un propose base sur le jeu de rôle dans l'enseignement de la langue française pour 
quelque compétences de la communication orale a la faculté de pédagogie de Minia . thèses de magister .université de Minia, faculté de pédagogie

\section{B) Ouvrages en langue française :}

12-Conseil de l'Europe (2001). Cadre européen commun de référence pour les langues : apprendre, enseigner, évaluer. Paris : Didier, 196p. en ligne: http://www.coa.int/t/dg4/linguistic/source/framework-fr.pdf

13- Crahay, M. (1999) . Psychologie de l'éducation. Paris. Presses universitaires de France.

14- La fortune, L \& Deaudelin, C . (2001). Accompagnement socioconstructiviste pour s'approprier une réforme en éducation Sainte Foy, Québec : presses de l'université du Québec.

15- PLANE, S. (2004) 'L'enseignement de l'oral : enjeux et évolution' in Cl. Garcia-Debanc et S. Plane (coord.) : Comment enseigner à l'école primaire ? Paris, Hatier Pédagogie, pp.11-38

16- Procher, L.(2004). L'enseignement des langues étrangères, Hachette ,paris

\section{c) Périodiques en langue française :}

17- Estelle, D. (2003). Comment favoriser la communication orale dans l'apprentissage d'une langues vivantes étrangères? En ligne http://.dijon.iufm.fr/doc/memoire/mes2003/3_0161078K.pdf.

18- Hafez, Hanan. (2003). Evaluation du traitement didactique de la communication orale en français langue étrangère chez les futurs- enseignants dans les facultés de pédagogie à la lueur de l'enseignement stratégique. L'association égyptienne des curricula et des méthodologies. Revue NO 152

19- Seyame, Wafaa, (2014). Developpement de l'expression orale chez les futurs enseignants par la perspective pctionnelle. $\mathrm{N}^{\circ} 204$ (Août), 49-100.

\section{2- Références en langue anglaise:}


20- Lerman, S. (2000). The social turn in mathematics education research. In J. Boaler (Ed.) Multiple Perspectives on Mathematics Teaching and Learning (pp. 19-44). Westport, CT: Ablex.

\section{Sitographie :}

21- Giffard,D.(1985)Dispensablesurhttp://www.khouasweb.12 3.fr/index.php/ressources/didactique/pedagogie/546-commentenseigner-lexpression-oral jeudi 1 septembre $2011 \mathrm{e}$

\section{Références en langue arabe :}

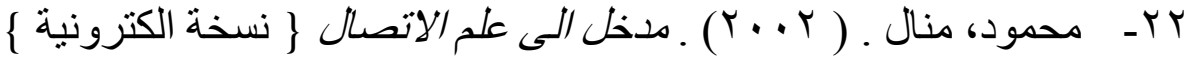

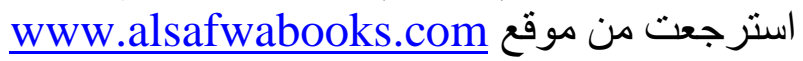

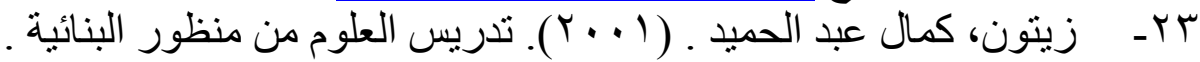

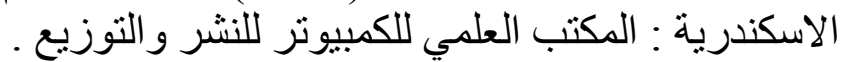

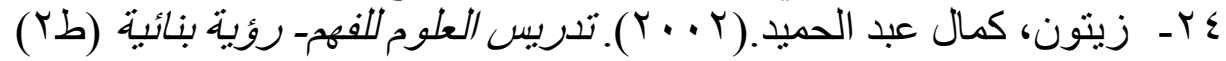

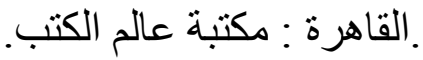

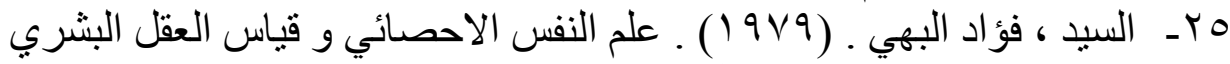

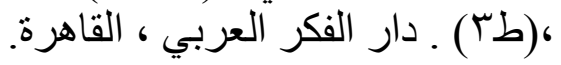

